\title{
Magnetic Anisotropy Engineering in Thin Film Ni Nanostructures by Magnetoelastic Coupling
}

\author{
S. Finizio, ${ }^{1}$ M. Foerster ${ }^{1,2}$ M. Buzzi, ${ }^{3}$ B. Krüger, ${ }^{1}$ M. Jourdan, ${ }^{1}$ C. A. F. Vaz, ${ }^{1,4}$ J. Hockel,,${ }^{5}$ T. Miyawaki, ${ }^{6}$ \\ A. Tkach, ${ }^{1}$ S. Valencia, ${ }^{7}$ F. Kronast, ${ }^{7}$ G. P. Carman, ${ }^{5}$ F. Nolting, ${ }^{3}$ and M. Kläui ${ }^{1, *}$ \\ ${ }^{1}$ Institut für Physik, Johannes Gutenberg Universität, Staudingerweg 7, Mainz, D-55128, Germany \\ ${ }^{2}$ ALBA Synchrotron Light Source, Carretera BP 1413, kilometer 3.3 Cerdanyola del Valles, E-08290, Spain \\ ${ }^{3}$ Swiss Light Source, Paul Scherrer Institut, Villigen, CH-5232, Switzerland \\ ${ }^{4}$ SwissFEL, Paul Scherrer Institut, Villigen, CH-5232, Switzerland \\ ${ }^{5}$ Department of Mechanical and Aerospace Engineering, University of California, \\ Los Angeles, California 90095, USA \\ ${ }^{6}$ Department of Crystalline Materials Science, Nagoya University, Nagoya, 464-8603, Japan \\ ${ }^{7}$ Helmholtz-Zentrum-Berlin für Materialien und Energie GmbH, Albert-Einstein Straße 15, \\ Berlin, D-12489, Germany
}

(Received 28 January 2014; revised manuscript received 28 February 2014; published 27 March 2014)

A phenomenon that can be exploited for the manipulation of magnetization without the conventional current-generated magnetic fields is magnetoelastic coupling, which might, thus, pave the way for lowpower data-storage devices. Here, we report a quantitative analysis of the magnetic uniaxial anisotropy induced by piezoelectric strain in Ni nanostructured squares. By applying strain, the magnetic domains in $\mathrm{Ni}$ nanostructured squares can be manipulated by the magnetoelastic effect in the Ni. The strain-induced anisotropy displaces the domain walls in the square leading to changes in the domain sizes. By comparing the experiments with micromagnetic simulations, the resulting uniaxial anisotropy is quantified. We find a good agreement for a magnetostrictive constant of $\lambda_{s}=-26 \mathrm{ppm}$, confirming a full strain transfer from the piezoelectric to the Ni nanostructures and the retainment of a bulklike $\lambda_{s}$.

DOI: 10.1103/PhysRevApplied.1.021001

Devices for spintronics applications are based on the manipulation of magnetization. Conspicuous examples include the control of the magnetization direction of ferromagnetic electrodes (e.g., in magnetic tunneling junctions for magnetic random access memory applications [1]) or of ferromagnetic domains in nanowires (for storage class memory, such as the racetrack memory [2]). Conventionally, this manipulation is achieved by a current flowing through a coil or stripline, which generates an Oersted field. Another approach that has recently become very popular is to exploit the spin-torque effect due to a transfer of angular momentum from a spin polarized charge current [3]. In particular, the writing of the magnetic information through spin torque requires large current densities, which lead to a high power consumption [4]. In general, such currents are always associated with unwanted heat dissipation in the device; therefore, the manipulation of the magnetization without the necessity to apply electrical currents is of strong interest for device applications. An encouraging approach to achieve low power dissipation is based on the manipulation of magnetic ordering by the application of electrical fields due to magnetoelectric coupling. Different mechanisms where the application of an electric field leads to a change of

*Corresponding author. klaeui@uni-mainz.de magnetic properties have been suggested and studied. One promising approach is to use piezoelectric substrates or thin films onto which ferromagnetic layers of specific materials, such as $\mathrm{Ni}$, are deposited. The piezoactive material generates a strain upon application of an electric field, inducing a strain in the magnetic film, which modifies the thin film crystal structure, and, by spin-orbit coupling, this changes the magnetic properties of the ferromagnetic material. Strain-induced magnetization changes due to the magnetoelastic coupling allow one to induce phase transitions, changes of the saturation magnetization, of the Curie temperature, and, most importantly, to manipulate the magnetic anisotropy [5-7]. The manipulation of the direction and strength of the uniaxial anisotropy is ideal to achieve the switching of the magnetization direction or to move domain walls, which constitute clear points of interest for applications in devices. As thin film nanostructures are required for device applications, special care needs to be taken for achieving the control of the magnetization in such nanoscale elements by magnetoelastic coupling.

Different piezoelectric materials can be used as substrates for ferromagnetic thin films. Key properties include the magnitude of static and dynamic strain, the compatibility with the growth of the magnetic thin films, and the possibility to induce strain in a desired direction. One example is $\left[\mathrm{Pb}\left(\mathrm{Mg}_{0.33} \mathrm{Nb}_{0.66}\right) \mathrm{O}_{3}\right]_{0.68}\left[\mathrm{PbTiO}_{3}\right]_{0.32}$ (PNMPT), which exhibits a pseudocubic perovskite crystal 
structure with a lattice parameter of $4.02 \AA[8]$ and which can be used to induce large strains in different directions depending on the crystalline orientation and resulting surface of the substrate $[8,9]$. Such substrate material was previously successfully utilized, for instance, by Herklotz et al. [10] for the generation of strain in manganite thin films. While manganites are interesting for their complex magnetic and electric properties, the relatively low Curie temperature (in particular, of the surface magnetization [11]) makes the use of this compound in applications challenging. More suitable materials for applications are $3 d$ metals, which exhibit reliable magnetic properties at room temperature. Moreover, if polycrystalline films can be utilized (as in the case of $3 d$ metals), this makes the materials compatible with widespread applications, but it is unclear if polycrystalline films exhibit the same large magnetostriction as the material in bulk form. A suitable material, which has been used as bulk in the past to study magnetoelastic coupling, is $\mathrm{Ni}[6,12]$. In fact, thin films of Ni have recently been investigated and first studies of Ni nanostructures show a clear influence of the strain on the magnetization configuration $[6,13]$. As both the strain relaxation in thin layers and the polycrystalline nature of the $\mathrm{Ni}$ can reduce the magnitude of the magnetoelastic effect, rendering this applications-relevant system unsuitable, a careful quantification of the effectively achievable magnitude of the magnetoelastic coupling needs to be carried out. This is a crucial step towards potential applications, as it is not clear how large the achievable magnetic anisotropy changes are, since strain relaxation in buffer layers at edges and at grain boundaries combined with a possibly reduced magnetostrictive constant for polycrystalline materials can limit the achievable magnetization changes.

In the work presented here, the strength of the magnetoelastic coupling in Ni nanostructures is determined by direct magnetic imaging in applied strain conditions. We show that the magnetization can be strongly modified by the induced strain even for moderate applied electric fields. By comparison with micromagnetic simulations, the induced anisotropy changes are evaluated, and it is found that, even for polycrystalline $\mathrm{Ni}$ on thick buffer layers, the magnetoelastic coupling is comparable to that found in bulk $\mathrm{Ni}$ showing negligible strain relaxation and no significant reduction in the magnetostrictive constant due to the polycrystalline nature of the $\mathrm{Ni}$, thus, demonstrating an applicability even for technologically relevant thin film nanostructures.

The investigations presented in this article are carried out on $2-\mu \mathrm{m}$-wide Ni squares. The samples are fabricated by depositing $35 \mathrm{~nm}$ of Ni by electron-beam evaporation on top of a 50-nm sputter-deposited buffer Pt electrode (with a 5-nm Ti adhesion layer) grown on a 0.5-mm-thick PMN-PT (011) substrate (Atom Optics Co., Ltd., Shanghai, China) in the as-received condition (i.e., no electrical poling prior to the deposition of the Ni). Patterning is carried out by electron-beam lithography employing a lift-off technique. Further details on the deposition procedure and on the fabrication of the nanostructures can be found in Ref. [6]. The average size of the polycrystalline grains determined by x-ray diffraction analyses on continuous films grown in the conditions used here is about $10 \mathrm{~nm}$. A schematic of the analyzed samples is shown in Fig. 1(a), where also a scanning electron microscopy image of a $2-\mu \mathrm{m}$-wide square structure is presented [Fig. 1(b)]. A thickness of $35 \mathrm{~nm}$ is chosen for the Ni films, as it is sufficient to allow for welldefined magnetic structures. For a thicker Ni film, one expects a lower strain transfer efficiency, so this particular thickness is chosen for the experiments described here.

Imaging of the magnetic configuration of the nanostructures is carried out by photoemission electron microscopy (PEEM) utilizing the $\mathrm{x}$-ray magnetic circular dichroism effect (XMCD) to obtain magnetic contrast [14]. The sample is illuminated by circularly polarized $\mathrm{x}$ rays tuned to the $\mathrm{L}_{3}$ edge of $\mathrm{Ni}(852.5 \mathrm{eV})$. All measurements are carried out at room temperature at the beam line UE-49-PGM-a at BESSY II, Berlin [15] and at the beam line SIM (X11MA) at the Swiss Light Source, Villigen [16], both equipped with an Elmitec PEEM setup (type LEEM III). Electric fields are applied to the piezoelectric material by a floating voltage generator inside the high voltage environment of the PEEM control unit; contacting is carried out as shown in Fig. 1(a). The sample

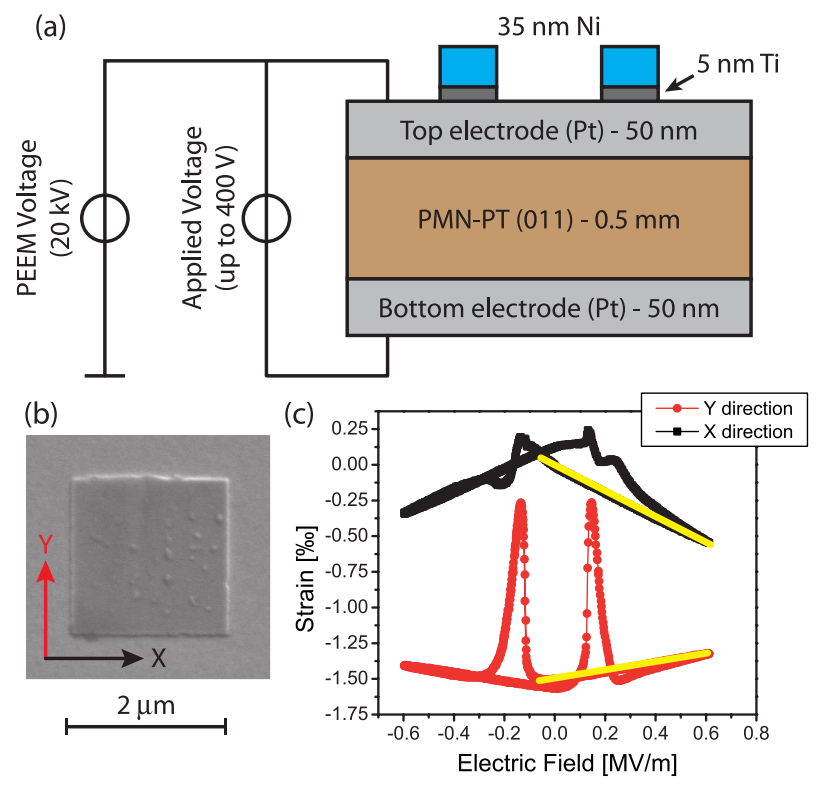

FIG. 1. (a) Schematic of the sample structure, including the electrical contact setup. (b) Scanning electron micrograph of a $2-\mu \mathrm{m}$-wide $\mathrm{Ni}$ square structure. Indicated are the $x$ and $y$ directions shown in (c). (c) Hysteresis cycle of the in-plane strain ( $x$ and $y$ directions) as a function of the applied out-of-plane electric field ( $z$ direction) of a PMN-PT (011) substrate. Adapted from Ref. [9]. The yellow line indicates the electric field range used in the experiments described here. 
is attached to the PEEM sample holder in a single location, using a silver colloidal solution. This particular arrangement allows for the free expansion of the substrate in all spatial directions, preventing any clamping, and, thus, allowing for the use of the full strain generated by the piezoelectric substrate.

For the analyses we present here, a particularly apt geometry is the high symmetry square for a broad range of lateral sizes and film thicknesses, as this geometry allows for the formation of the simple magnetic flux-closure Landau state [17], where four equally sized domains are formed due to shape anisotropy, as shown in Fig. 2. As the anisotropy is changed, the size of the magnetic domains changes and domains with the magnetization pointing along an easy axis grow, while perpendicular domains with magnetization along the hard axis shrink. Thus, the size of the domains, which can be experimentally measured by magnetic microscopy, acts as an indicator of the magnitude of the anisotropies applied to the magnetic material.

The PMN-PT is oriented in the (011) direction, which enables the generation of an in-plane strain by applying an out-of-plane electric field $[8,9]$. The piezoelectric strain behaves hysteretically with respect to the applied electric field, as shown in Fig. 1(c). However, after an initial poling of the piezoelectric material (the substrate is initialized with a field of $0.6 \mathrm{MV} \mathrm{m}^{-1}$ ), it is possible to achieve a linear nonhysteretic behavior by restricting the applied electric field to a range in which the polarization of the substrate is not reversed. Fields between -0.1 and $0.6 \mathrm{MV} \mathrm{m}^{-1}$ [as marked in Fig. 1(c), where a positive field corresponds to a positive voltage applied to the bottom electrode with respect to the top electrode] are applied during the experiments we present here. A concern is the influence of the 50-nm-thick Pt buffer layer, which can reduce the strain transfer from the substrate to the Ni nanostructures. While any shear lag effect in the nanostructures can be expected to

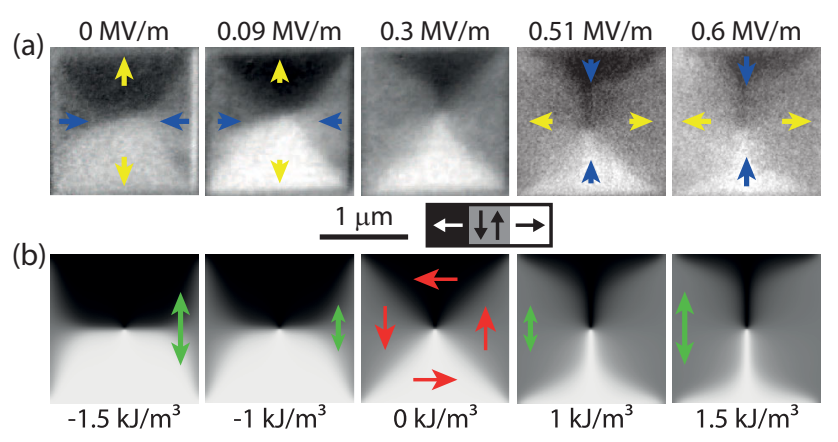

FIG. 2. (a) XMCD-PEEM images of a $2-\mu \mathrm{m}$-wide Ni square, at different applied electric fields. The blue and yellow arrows indicate the directions of the compressive and tensile strains, respectively. (b) Micromagnetic simulations of a $2-\mu$ m-wide $\mathrm{Ni}$ square, assuming different uniaxial anisotropies. The gray scale bar and the red arrows indicate the direction of the magnetic contrast, while the green arrow indicates the direction of the uniaxial anisotropy applied in the micromagnetic simulations. extend laterally only for a distance corresponding to approximately 2-3 times the thickness into the nanostructure, strain relaxation in the electrode material can play a role, due to the possible formation of defects in the material caused by the application of the piezoelectric strain.

For the geometry we use here, the magnetic ground state is the Landau state. However, due to the finite probability of electrical discharges naturally inherent to the PEEM setup, which lead to strong magnetic fields on the sample, it is also possible to nucleate more complex metastable states due to electric discharges in the PEEM. In this case, the Landau state [17] is recovered by demagnetizing the system by applying alternating magnetic fields with an in situ electromagnet.

To study the induced magnetic anisotropies, the magnetic configuration of the Ni square is imaged as a function of the electric field applied to the substrate, i.e., for different strain conditions, as shown in Fig. 2(a). The central image in Fig. 2(a) is showing a symmetric Landau state, which corresponds to the unstrained, shape-anisotropy dominated state. With the application of strain, two domains (with antiparallel magnetization) grow in size, while the other two with magnetization perpendicular to the other two domains shrink. Domains with magnetization parallel to the applied compressive strain (indicated by the blue arrows) grow in size at the expense of the domains with perpendicular magnetization (parallel to the tensile strain indicated by the yellow arrows). This is direct evidence that an uniaxial anisotropy is induced in the magnetic material upon the application of piezoelectric strain. The observed behavior is qualitatively in line with the expected behavior of $\mathrm{Ni}$, which, given the negative sign of its magnetostrictive constant (in bulk Ni, $\lambda_{s} \simeq-32 \mathrm{ppm}$ [18]) should, indeed, present a growth of the magnetic domains pointing along the direction of the compressive strain.

Next, the induced anisotropies are quantified. To conduct this analysis, the size of the domains with magnetization parallel or perpendicular to the applied compressive strain direction is determined as a function of the applied electric field (i.e., piezoelectric strain), as shown in Fig. 3. Corresponding micromagnetic simulations of a $2-\mu \mathrm{m}$-wide $\mathrm{Ni}$ square with different strain-induced anisotropies in addition to the contribution of shape anisotropy are then carried out to find for which anisotropy values the domain configurations agree.

The simulations are carried out utilizing the MicroMagnum framework [19], with the following parameters for $\mathrm{Ni}$ : exchange constant $10^{-11} \mathrm{~J} \mathrm{~m}^{-1}$ and saturation magnetization $2.1 \times 10^{5} \mathrm{~A} \mathrm{~m}^{-1}$ determined by superconducting quantum interference device magnetometry on a continuous Ni film of similar characteristics. These parameters lead to an exchange length of approximately $19 \mathrm{~nm}$. The numerical discretization utilized for the calculations presents a cell size of $8 \mathrm{~nm}$ in the in-plane direction and 


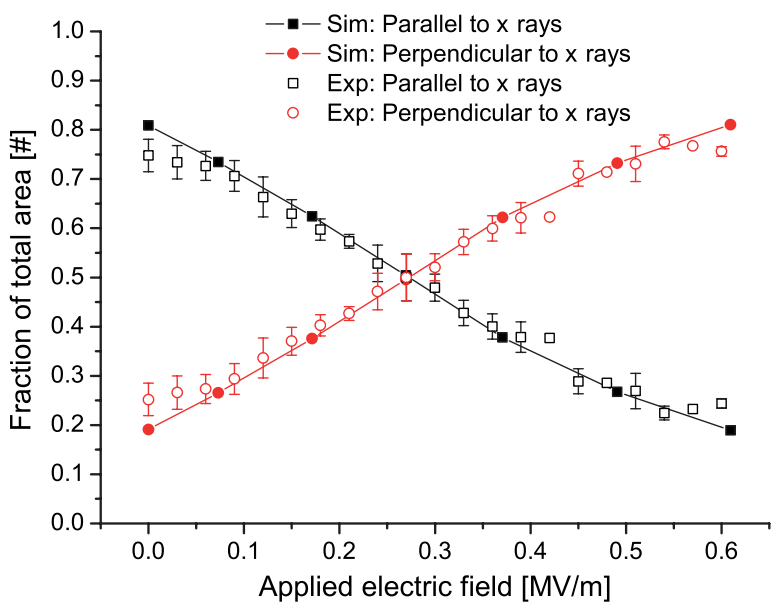

FIG. 3. Fraction of the area occupied by domains with magnetization parallel (black square symbols) and perpendicular (red circle symbols) to the x-ray beam (i.e., size of black and white and grey areas in the images). The empty squares and circles indicate data points determined from the experimentally obtained images; the full squares and circles indicate data points determined from the simulated images. The lines are guides to the eye.

$35 \mathrm{~nm}$ in the out-of-plane direction (the total thickness of the structure is $35 \mathrm{~nm}$ ). The fact that the determined agreement is similarly good everywhere in the nanostructure allows one to observe that possible shear lag effects at the edges of the nanostructure are not significantly altering the magnetic properties.

The simulations are carried out for a range of anisotropies, as shown in Fig. 2(b), to simulate the contribution of the applied piezoelectric strain, checking the validity of the simulations by comparing the simulated configurations and XMCD-PEEM images of the Ni square in the symmetric Landau flux-closure state as a function of an externally applied magnetic field, which provide a benchmark that can be easily simulated micromagnetically. The comparison between the simulations and the experimental images acquired with different applied magnetic field yields a good agreement for the parameters given above. In order to directly compare the experimental images with the micromagnetic simulations, the simulated uniaxial anisotropy is converted to an effective applied strain by using the following relation [20]:

$$
K_{\mathrm{ME}}=-\frac{3}{2} \lambda_{s} Y\left|\varepsilon_{x}-\varepsilon_{y}\right|,
$$

where $K_{\mathrm{ME}}$ denotes the magnetoelastic anisotropy coefficient, $\lambda_{s}$ the magnetostrictive constant of $\mathrm{Ni}, Y$ the Young's modulus of $\mathrm{Ni}$, and $\left|\varepsilon_{x}-\varepsilon_{y}\right|$ the in-plane strain difference. A value of $220 \mathrm{GPa}$ is utilized for the Young's modulus, which corresponds to the value measured in bulk Ni [21]. The adoption of the bulk value for the Young's modulus constitutes an approximation for the analyzed material. However, as the size of the grains is small (approximately $10 \mathrm{~nm}$ ) compared to the size of the structures, using the bulk value for the Young's modulus constitutes a reasonable approximation. The value of $\left|\varepsilon_{x}-\varepsilon_{y}\right|$ is then converted to an effective applied electric field by using the strain-vs-field curve of PMN-PT (011).

Because of the polycrystalline nature of the Ni (i.e., no effective magnetocrystalline anisotropy is present due to the random orientation of the many grains), the micromagnetic simulations do not include any additional anisotropy contribution besides the one arising from the applied strain. Note that the zero strain condition at which the magnetic state of the Ni square falls into a symmetric Landau state is achieved with an applied electric field of approximately $0.27 \mathrm{MV} \mathrm{m}^{-1}$. Since there is no further externally applied electric fields to induce a strain, this implies that a preexisting anisotropy is present. This may be related to the thin film deposition on the PNM-PT substrate without prior poling, i.e., on a substrate with different ferroelectric domains resulting in regions with different prestrain after poling [13]. As the Ni grows polycrystalline on top of the $\mathrm{Pt}$ electrode layer, a growth-induced anisotropy resulting from the lattice mismatch at the interface is not expected. However, a possible origin of the preexisting anisotropy can be due to microscopic step formation on the substrate surface due to a miscut.

Thus, the zero in $\left|\varepsilon_{x}-\varepsilon_{y}\right|$ of Eq. (1) is shifted to the value of the strain condition at which the unperturbed Landau state is observed experimentally. Such an unperturbed Landau state results from zero strain and zero anisotropy (with the exception of shape anisotropy), and, thus, allows for the identification of the strain-free condition. With this adjustment, the experimental data fit well with the simulations with a magnetostrictive constant $\lambda_{s}=-26 \mathrm{ppm}$, which is within the range typically found for bulk Ni.

In the analyzed electric field range, the fraction of the total area occupied by the magnetic domains pointing along the compressive strain direction scales almost linearly with the applied field. For larger field values, we observe deviations from the linear behavior, due to the increasing importance of the shape anisotropy contribution, which counteracts the induced anisotropy. The simulations show that, for high strains (not achievable experimentally due to cracking of the substrate which occurs at high applied piezoelectric strains), the dimensions of the domain walls pointing along the compressive strain direction saturate, due to the dominant role of the shape anisotropy.

The value of the magnetostrictive constant, which is found from the simulations, is about $10 \%$ smaller than the bulk value. This might be due to strain relaxation in the buffer layer and to the polycrystalline nature of the deposited material but shows that a very sizeable magnetoelastic coupling can be obtained. 
Finally, as the substrate exhibits a $\left[\mathrm{PbTiO}_{3}\right]_{x}$ composition of $x \simeq 0.32$, a mixture between monoclinic and tetragonal phases may occur in the substrate [22]. Such phase mixture can lead to the formation of strain inhomogeneities at the substrate surface, which, in turn, can result in an inhomogeneous magnetic behavior of the $\mathrm{Ni}$ nanostructures. However, as the observed magnetic structure for the $\mathrm{Ni}$ squares closely matches the simulations (which are carried out under the assumption of a homogeneous strain condition), any major influence on the magnetic configuration of the Ni squares due to strain inhomogeneities can be ruled out.

In conclusion, we achieve a manipulation of the magnetic configuration of $\mathrm{Ni}$ nanostructured squares without any electrical currents by applying only electrical fields to a piezosubstrate. We achieve the manipulation of the magnetic configuration by the exploitation of the magnetoelastic effect, which originates from the strain induced by the piezoelectric substrate of the nanostructures. We find that the domain walls of a magnetic square in the Landau state can be reversibly displaced by the induced anisotropy leading to a change in the domain size. Combining the experimental results with micromagnetic simulations, we determine the resulting uniaxial anisotropy quantitatively. We find a good quantitative agreement for a magnetostrictive constant of the Ni nanostructures of $\lambda_{s}=-26 \mathrm{ppm}$, which is only slightly smaller than the bulk value of $\mathrm{Ni}$. The calculated value of the magnetostrictive constant can be explained only if the strain transfer from the substrate to the nanostructures across the buffer layer occurs homogeneously with negligible relaxation effects and if the polycrystalline Ni shows a magnetostrictive constant comparable to that of the single crystalline Ni. This shows that even for thick buffer layers and polycrystalline films, large effects can be obtained for these applications' relevant nanostructures.

Part of this work was performed at the Swiss Light Source, Paul Scherrer Institut, Villigen, Switzerland and at the Helmholtz-Zentrum-Berlin, Germany. The authors further acknowledge the financial support by the EU's 7th Framework Programme IFOX (Grant No. NMP3-LA2010 246102), the European Research Council through the Starting Independent Researcher Grant MASPIC (Grant No. ERC-2007-StG 208162), the National Science Foundation under Grant No. 1160504 NSF Nanosystems Engineering Research Center for Translational Applications of Nanoscale Multiferroic Systems (TANMS), the Swiss National Science Foundation, the Graduate School of Excellence "Materials Science in Mainz" (Grant No. GSC 266), and the Deutsche Forschungsgemeinschaft (DFG).

[1] J. Åckereman, Toward a universal memory, Science 308, 508 (2005).
[2] S. Parkin, M. Hayashi, and L. Thomas, Magnetic domainwall racetrack memory, Science 320, 190 (2008).

[3] J. A. Katine, F. J. Albert, R. A. Buhrman, E. B. Myers, and D. C. Ralph, Current-driven magnetization reversal and spin-wave excitations in $\mathrm{Co} / \mathrm{Cu} / \mathrm{Co}$ pillars, Phys. Rev. Lett. 84, 3149 (2000).

[4] K. Wang, J. Alzate, and P. Khalili Amiri, Low-power nonvolatile spintronic memory: STT-RAM and beyond, J. Phys. D 46, 074003 (2013).

[5] M. Liu, J. Hoffman, J. Wang, J. Zhang, and B. NelsonCheeseman, Non-volatile ferroelastic switching of the Verwey transition and resistivity of epitaxial $\mathrm{Fe}_{3} \mathrm{O}_{4}$ /PMN-PT (011), Sci. Rep. 3, 1876 (2013).

[6] J. L. Hockel, A. Bur, T. Wu, K. P. Wetzlar, and G. P. Carman, Electric field induced magnetization rotation in patterned Niring $/\left[\mathrm{Pb}\left(\mathrm{Mg}_{1 / 3} \mathrm{Nb}_{2 / 3}\right) \mathrm{O}_{3}\right]_{(1-0.32)}-\left[\mathrm{PbTiO}_{3}\right]_{0.32}$ heterostructures, Appl. Phys. Lett. 100, 022401 (2012).

[7] C. Adamo, X. Ke, H. Q. Wang, H. L. Xin, T. Heeg, M. E. Hawley, W. Zander, J. Schubert, P. Schiffer, D. A. Muller, L. Maritato, and D. G. Schlom, Effect of biaxial strain on the electrical and magnetic properties of (001) LSMO thin films, Appl. Phys. Lett. 95, 112504 (2009).

[8] K. Uchino, Relaxor ferroelectric devices, Ferroelectrics 151, 321 (1994).

[9] T. Wu, P. Zhao, M. Bao, A. Bur, J. L. Hockel, K. Wong, K. P. Mohanchandra, C.S. Lynch, and G. P. Carman, Domain engineered switchable strain states in ferroelectric $\left[\mathrm{Pb}\left(\mathrm{Mg}_{1 / 3} \mathrm{Nb}_{2 / 3}\right) \mathrm{O}_{3}\right]_{(1-x)}-\left[\mathrm{PbTiO}_{3}\right]_{x}(\mathrm{PMN}-\mathrm{PT}, x \simeq 0.32)$ single crystals, J. Appl. Phys. 109, 124101 (2011).

[10] A. Herklotz, J. D. Plumhof, A. Rastelli, O. G. Schmidt, L. Schultz, and K. Dörr, Electrical characterization of PMN-28\%PT(001) crystals used as thin-film substrates, J. Appl. Phys. 108, 094101 (2010).

[11] R. M. Reeve, C. Mix, M. König, M. Foerster, G. Jakob, and M. Kläui, Magnetic domain structure of $\mathrm{La}_{0.7} \mathrm{Sr}_{0.3} \mathrm{MnO}_{3}$ thin-films probed at variable temperature with scanning electron microscopy with polarization analysis, Appl. Phys. Lett. 102, 122407 (2013).

[12] A. Bur, T. Wu, J. L. Hockel, C.-J. Hsu, H. K. D. Kim, T.-K. Chung, K. Wong, K. L. Wang, and G. P. Carman, Straininduced magnetization change in patterned ferromagnetic nickel nanostructures, J. Appl. Phys. 109, 123903 (2011).

[13] M. Buzzi, R. V. Chopdekar, J. L. Hockel, A. Bur, T. Wu, N. Pilet, P. Warnicke, G. P. Carman, L. J. Heyderman, and F. Nolting, Single domain spin manipulation by electric fields in strain coupled artificial multiferroic nanostructures, Phys. Rev. Lett. 111, 027204 (2013).

[14] J. Stöhr, Y. Wu, B. D. Hermsmeier, M. G. Samant, G. R. Harp, S. Koranda, D. Dunham, and B. P. Tonner, Elementspecific magnetic microscopy with circularly polarized X-rays, Science 259, 658 (1993).

[15] F. Kronast, J. Schlichting, F. Radu, S. K. Mishra, T. Noll, and H. A. Durr, Spin-resolved photoemission microscopy and magnetic imaging in applied magnetic fields, Surf. Interface Anal. 42, 1532 (2010).

[16] U. Flechsig, F. Nolting, A. Fraile-Rodriguez, J. Krempasky, C. Quitmann, T. Schmidt, S. Spielmann, and D. Zimoch, Performance measurements at the SLS SIM beamline, AIP Conf. Proc. 1234, 319 (2010). 
[17] A. Hubert and R. Schäfer, Magnetic Domains: The Analysis of Magnetic Microstructures (Springer-Verlag, Berlin, 1998).

[18] R. Cullity and C. D. Graham, Introduction to Magnetic Materials (Wiley-IEEE Press, New York, 2008).

[19] MicroMagnum, http://micromagnum.informatik.unihamburg.de.

[20] M. Weiler, A. Brandlmaier, S. Geprägs, M. Althammer, M. Opel, C. Bihler, H. Huebl, M. S. Brandt, R. Gross, and S. T.

B. Goennenwein, Voltage controlled inversion of magnetic anisotropy in a ferromagnetic thin film at room temperature, New J. Phys. 11, 013021 (2009).

[21] H. M. Ledbetter and R. P. Reed, Elastic properties of metals and alloys, 1. Iron, nickel and iron-nickel alloys, J. Phys. Chem. Ref. Data 2, 531 (1973).

[22] B. Noheda, D. E. Cox, G. Shirane, J. Gao, and Z.-G. Ye, Phase diagram of the ferroelectric relaxor $(1-x) \mathrm{PbMg}_{1 / 3} \mathrm{Nb}_{2 / 3} \mathrm{O}_{3}-x \mathrm{PbTiO}_{3}$, Phys. Rev. B 66, 054104 (2002). 